\title{
Delivery Vehicles for Antiplaque Agents
}

\section{Siddharth $\mathrm{T}^{*}$}

Department of Periodontology and Oral Implantology, ITS Dental College, Muradnagar; Ghaziabad, India

\begin{abstract}
Oral health is an integral part of general health. Poor oral health can have adverse effects on general health. Hence a good oral and general health is very essential, which in turn is achieved by good oral hygiene. Even though, the concept of good oral hygiene evolved some 5000 yrs back. It is only in the beginning of the $19^{\text {th }}$ century it gained more importance. The earlier concept of maintaining oral hygiene by just cleaving the food debris has been taken over by removal of plaque, an infectious agent responsible for many common dental diseases, in the $20^{\text {th }}$ century. Plaque control is one of the keystones of the practice of dentistry, without it, oral health can neither be attained, nor preserved. For patient with healthy periodontium, Plaque control means preservation of health. For the patient with periodontal disease, it means optimal healing, following treatment. For the patient with treated periodontal disease, plaque control means the prevention of recurrence of disease.
\end{abstract}

Keywords: Plaque; Periodontium; Antiplaque agents; Humectants; Dentistry

\section{Introduction}

Because interdentally and sub gingival sites are relatively inaccessible to mouth-rinsing, they necessitate alternative methods of application of anti plaque chemicals. A variety of home care aids, including brushes, floss and tooth picks have been used to apply antiplaque agent interdentally. Morgh and Bjorvan used toothpicks to carry sodium and amine fluoride to the tooth surface and Newbrun found that iodine impregnated floss reduced the proportion of streptococcus mutans in approximal plaque more than plain floss. Stannous fluoride containing floss has been shown to reduce bacterial growth chlorhexidine impregnanted floss was found to produce greater decrease in plaque than untreated floss.

\section{Mouth rinse}

They are the simplest and most common delivery system for antiplaque agents. This vehicle is generally a mixture of water and alcohol to which flavour, surfactant, humectants are added to improve cosmetic properties. The more potent and clinically relevant surfactants include: bisbiguanides, particularly chlorhexidine, quanternaary ammonium salts, aliphatic alcohol and sanguinarine. such compound may exert their effect by direct injury to bacteria by electrostatic, hydrophobic interactions with bacterial enzymes.

Mouth rinses can be divided into 2 major categories:

- First generation : Mouthrinses capable of reducing plaque to about $20-50 \%$ when used to $4-5$ times a day and have limited or no substantivity. e.g. listerine

- Second generation: They are capable of reducing plaque by $70-90 \%$ when used 1-2 times a day and have an effective substantivity lasting 12-18 hours or longer. e. g. chlorhexidine.

\section{Properties of mouth rinses}

- Have an antimicrobial spectrum appropriate for site of application.

- Be rapidly effective against microorganism.

- Be able to directly kill or inactivate suspectible organism

- Not induce hypersensitivity with repeated applications
- Have residual effect after each use

- Be reasonable cost for routine use.

\section{Dentrifices}

Are widely accepted as part of daily oral hygiene practices and have proved successful for the delivery of fluoride. The basic components of dentrifices include humectants, flavourings agents, binders and abrasives.

\section{Dental gel}

Gels for the delivery of antiplaque agents are usually simple, thickened, aqueous systems containing humectants with neither abrasive nor foaming agent. As such it is compatible with most antiplaque agents. Hansen found chlorhexidine gel to have little effect on plaque and none on gingivitis. However chlorhexidine gel was found to be effective in plaque control in handicapped children.

\section{Sprays}

Sprays have the advantage of focusing delivery on the required site. The dose is clearly reduced and for antiseptics such as chlorhexidine this has taste advantages. Chlorhexidine sprays were found particularly useful for plaque control in physically and mentally handicapped groups.

\section{Chewing gum}

Over a relatively short period there has been interest in employing chewing gum to deliver a variety of agents for oral health benefits. Chewing gums alone appear to have little in the way of plaque control benefits particularly at sites prone to gingivitis [1]. Nonetheless, the vehicle has been used to deliver chemical agents such as chlorhexidine and, when used as an adjunct to normal tooth brushing, reduced plaque

*Corresonding author: Siddharth, Post-Graduate Scholar, Department of Periodontology and Oral Implantology, ITS Dental College, Muradnagar Ghaziabad, India, Tel: +919654086903; E-mail: dr.siddharthtevatia@gmail.com

Received June 30, 2017; Accepted July 05, 2017; Published July 15, 2017

Citation: Siddharth T (2017) Delivery Vehicles for Antiplaque Agents. J Formul Sci Bioavailab 1: 112

Copyright: (c) 2017 Siddharth T. This is an open-access article distributed unde the terms of the Creative Commons Attribution License, which permits unrestricted use, distribution, and reproduction in any medium, provided the original author and source are credited. 
and gingivitis levels have been shown. The advantage with chewing gum is that usually kept in the mouth for longer time than rinses and toothpastes.

\section{Irrigators}

Irrigators were designed to spray water, under pressure, around the teeth. As they only removed debris, with little effect on plaque deposits. Antiseptics and other chemical plaque control agents, such as chlorhexidine, have been added to the reservoir of such devices.

\section{Varnishes}

Varnishes have been employed to deliver antiseptics including chlorhexidine, but the purpose has been to prevent root caries rather than as a reservoir for plaque control throughout the mouth.

\section{Slow release devices}

Recently there have been several attempts to use containers to apply antiplaque agents to periodontal pocket. The main aim to produce devices from which the antimicrobial may be released slowly releasing in a prolonged effect on plaque .These devices are polymer based matrices, generally containing an antiseptic or antibiotic. They are characterized according to matrix type as diffusion or chemically controlled system [2]. Soskone have shown that chlorhexidine release can be sustained from days upto months for a range of ethyl cellulose based devices

\section{Ideal Properties of an Antiplaque Agent}

1. Should eliminate only the pathogenic bacteria.

2. Prevent development of resistant bacteria.

3. Exhibit substantivity i.e. ability of an agent to bind to tissue surfaces and to be released over time to deliver an adequate dose of active principal ingredient the agent carries. (CHX gets adsorbed to oral tissues).

4. Safe to oral tissues at concentrations and dosages recommended.

5. Significantly reduce and gingivitis and prevent colonization of microorganisms.

6. Should not stain the teeth or alter taste.

\section{Substantivity}

Requires that the agent remain in contact with the tissues for long enough to be effective when applied to diseased sites. For an agent to be efficacious in the oral cavity, it must be substantive i.e. it must bind to tissue surface and be released over time to provide sustained activity against bacteria attempting to colonize the tooth surface $[3,4]$.

\section{Efficacy}

Each chemotherapeutic agent has to have bactericidal features if it is to be successful chemical plaque control. The agent to be effective against specific organisms causing oral diseases, such as dental caries, gingivitis and periodontitis $[5,6]$.

\section{Concentration}

Requires that the agent be specifically active against pathogenic bacteria and be effective against normal commensal oraganisms in the oral cavity.

\section{Specificity}

Requires that the agent be specifically active against pathogenic bacteria and be ineffective against normal commensal organisms in the oral cavity.

\section{Safety}

Requires that the agent may be used in the oral cavity without any detrimental effects to the tissues and also that the material be stable at normal room temperatures and not degrade spontaneously over a reasonable period of time

\section{Conclusion}

Mechanical plaque control is the mainstay for prevention of oral diseases, but it requires immense patient cooperation and motivation; therefore, chemical plaque control agents act as useful adjuvant for achieving the desired results. Antimicrobial and antiplaque agents in dentifrices and oral rinses act in several ways to reduce or remove dental biofilms and inhibit bacterial growth. Hence, it is imperative for the clinician to know the effect of these agents so that they can provide the patients with tailor-made prescription and prevent plaque-induced dental diseases.

\section{References}

1. Addy M, Moran JM (1997) Evaluation of oral hygiene products: science is true don't be misled by the facts: Perio 2000 15: 40-51.

2. Newman HN (1986) Modes of application of anti-plaque chemicals. J Clin Periodontol 13: 965-974.

3. Busscher HJ, Evans LV (1999) Oral biofilms and plaque control. HarwoodAcademic Publishers.

4. Lang NP, Lindhe J, Karring T (2008) Clinical Periodontology and Implant Dentistry (5thedn), p: 1448.

5. Clay B (2004) Walker: chemotherapeutics: antibiotics and other antimicrobials Perio 2000 36: 146-165.

6. Peter S (2008) Essentials of Preventive and Community Dentistry (2ndedn) 12: $447-458$ 